\title{
ON CYCLIC VECTORS OF WEIGHTED SHIFTS
}

\section{RABINDRANATHAN}

Abstract. Sufficient conditions on a sequence are given in order that the linear manifold spanned by its right translates is dense in certain Hilbert spaces of sequences.

1. Introduction. A vector $x$ in a Hilbert space $H$ is said to be a cyclic vector for a bounded operator $T$ on $H$ if the sequence $x, T x, T^{2} x, \cdots$ spans $H$. The search for cyclic vectors for certain operators leads to interesting approximation problems in function theory. One such operator is the forward shift in the space of one-sided sequences square-summable with respect to a weight. We consider a weight sequence $\{\rho(n)\}, n=0$, $1, \cdots$, satisfying the conditions

$$
\begin{gathered}
\rho(n)>0 \\
\sup _{n} \frac{\rho(n+1)}{\rho(n)}<\infty .
\end{gathered}
$$

Let $l^{2}(\rho)$ be the Hilbert space of all one-sided sequences $\left\{a_{n}\right\}$ for which the norm

$$
\|a\|=\left[\sum_{n=0}^{\infty}\left|a_{n}\right|^{2} \rho(n)\right]^{1 / 2}
$$

is finite. The shift operator $S$ on $l^{2}(\rho)$ is defined by

$$
S\left(a_{0}, a_{1}, \cdots\right)=\left(0, a_{0}, a_{1}, \cdots\right) .
$$

If (2) is satisfied, the operator $S$ is bounded and

$$
\|S\|=\sup [\rho(n+1) / \rho(n)]^{1 / 2} .
$$

(See [5, Chapter 10].)

The problem of deciding whether a vector $a$ in $l^{2}(\rho)$ is a cyclic vector for $S$ is the problem of deciding whether the right translates of $a$ span the

Presented to the Society, January 25, 1973; received by the editors August 15, 1972 and, in revised form, June 30, 1973.

AMS (MOS) subject classifications (1970). Primary 47B99, 46E20, 30A78; Secondary 47A60.

Key words and phrases. Cyclic vector, weighted sequence space, shift operator, outer function.

(c) American Mathematical Society 1974 
space $l^{2}(\rho)$. Motivated by classical results in harmonic analysis we introduce the "Laplace transform" of the sequence $a$ and try to link the cyclicity of $a$ to properties of its transform. (See the introduction of [3].) Let us assume that the number

$$
R=\lim \inf \rho(n)^{1 / 2 n}
$$

is different from zero. For each $a$ in $l^{2}(\rho)$ define the function $f_{a}$ by the relation

$$
f_{a}(z)=\sum_{n=0}^{\infty} a_{n} z^{n}
$$

Then $f_{a}$ is analytic in the disk $\{z:|z|<R\}$. The nonvanishing of $f_{a}$ is easily seen to be a necessary condition for the cyclicity of $a$.

In [2] Beurling gave a complete description of cyclic vectors for the forward shift in the case in which $\rho(n)=1$ for every $n$. It was proved there that a vector $a$ is cyclic if and only if the associated analytic function is outer. We must also mention the papers of Shapiro [9], [10], [11] where the problem is studied in weighted sequence spaces. The weight sequences that Shapiro considers are essentially moment sequences of measures on the unit interval. In that case the problem of the density of linear combinations of translates of a sequence becomes a problem of polynomial approximation in a weighted $L^{2}$ space. The main feature of the present work is that we do not assume that the weight sequence is a moment sequence.

2. The main result. Before we state the main result of this paper, we have to introduce some more notation. $H^{\infty}$ will denote the algebra of all bounded analytic functions in the unit disk. Let $N$ be the Nevanlinna class of analytic functions of bounded characteristic in the unit disk. $\mathrm{N}^{+}$ denotes the subclass of $N$ consisting of those functions $f$ for which

$$
\int_{0}^{2 \pi} \log ^{+}\left|f\left(r e^{i \theta}\right)\right| d \theta \rightarrow \int_{0}^{2 \pi} \log ^{+}\left|f\left(e^{i \theta}\right)\right| d \theta
$$

as $r \rightarrow 1-$, where $f\left(e^{i \theta}\right)=\lim _{r \rightarrow 1} f\left(r e^{i \theta}\right)$. It is well known that a function $f$ analytic in the unit disk belongs to the class $N^{+}$if and only if it is expressible in the form $f=\varphi / \psi$ where $\varphi$ and $\psi$ are in $H^{\infty}$ and $\psi$ is outer. (See $[4$, p. 26].)

THEOREM 1. Let $\{\rho(n)\}, n=0,1,2, \cdots$, be a nonincreasing sequence of positive numbers satisfying the condition

$$
\lim \inf \rho(n)^{1 / 2 n}=1 .
$$


Let $a=\left(a_{0}, a_{1}, \cdots\right)$ be an element of $l^{2}(\rho)$ and let $f_{a}$ be the analytic function defined by (4). If $1 / f_{a}$ is in the class $N^{+}$, then a is a cyclic vector for the forward shift on $l^{2}(\rho)$.

Remark. Condition (5) of the theorem deserves some comment. By elementary arguments, it can be shown that

$$
\lim \inf \rho(n)^{1 / n} \leqq \sup (\rho(\dot{n}+1) / \rho(n)) .
$$

The theorem can actually be proved under the assumptions:

(i) $\{\rho(n)\}$ is nonincreasing.

(ii) $\lim \inf \rho(n)^{1 / n}=\sup (\rho(n+1) / \rho(n))$.

(iii) The function $1 / f_{a}$ is in the class $N^{+}$in the disk $\{z:|z|<R\}$, where $R$ is defined by (3).

In the version that is stated above we have thus made the normalization that both the numbers involved in (ii) are equal to one. It should also be noted that if (ii) holds, then the lim inf in (ii) can be replaced by the limit.

We need the following lemma in the proof of the theorem. This lemma is of some independent interest. It must be compared with Lemma 5 of Shields and Wallen [12]. We must mention that in the proof of the lemma we do not assume that lim inf $\rho(n)^{1 / n}>0$.

LemMa. Let $\{\rho(n)\}, n=0,1,2, \cdots$, be a nonincreasing sequence of positive numbers. Let $\alpha=\left(\alpha_{0}, \alpha_{1}, \cdots\right)$ be the sequence of Fourier coefficients of an element $\varphi$ of $H^{\infty}$. Then the mapping $M_{\varphi}$ defined on $l^{2}(\rho)$ by $M_{\varphi} a=$ $\alpha * a$ is a bounded linear transformation. (Here $\alpha *$ a denotes the convolution of $\alpha$ with a.) The mapping $\varphi \rightarrow M_{\varphi}$ has the following properties.

(i) $\left\|M_{\varphi}\right\| \leqq\|\varphi\|_{\infty}$, where $\|\varphi\|_{\infty}$ is the supremum norm of $\varphi$ in $H^{\infty}$.

(ii) If $\left\{\varphi_{n}\right\}$ is a sequence in $H^{\infty}$ such that $\left\{\left\|\varphi_{n}\right\|_{\infty}\right\}$ is bounded and $\varphi_{n}\left(e^{i \theta}\right) \rightarrow \varphi\left(e^{i \theta}\right)$ for a.e. $\theta$ in $[0,2 \pi)$ and $\varphi$ is in $H^{\infty}$, then $\left\{M_{\varphi_{n}}\right\}$ converges to $M_{\varphi}$ in the strong operator topology.

Proof. This lemma will be deduced from Theorem 1.1 of [7] using a duality argument. Let $\tau(n)=1 / \rho(n)$. Then $\{\tau(n)\}$ is a nondecreasing sequence and the dual space of $l^{2}(\rho)$ can be identified with the space $l^{2}(\tau)$. The duality is given by

$$
\langle a, b\rangle=\sum_{n=0}^{\infty} a_{n} \bar{b}_{n}, \quad a \in l^{2}(\rho) \text { and } b \in l^{2}(\tau) .
$$

The adjoint of the forward shift $S$ on $l^{2}(\rho)$ is the backward shift $B$ on $l^{2}(\tau)$ given by $B\left(b_{0}, b_{1}, \cdots\right)=\left(b_{1}, b_{2}, \cdots\right)$.

The mapping that sends a sequence $b$ in $l^{2}(\tau)$ to the analytic function $\sum_{n=0}^{\infty} b_{n} z^{n}$ in the unit disk establishes an isometric isomorphism between 
$l^{2}(\tau)$ and the space $H^{2}(\tau)$ consisting of all analytic functions in the Hardy class $H^{2}$ whose Fourier coefficients are in $l^{2}(\tau)$. We shall use the spaces $l^{2}(\tau)$ and $H^{2}(\tau)$ interchangeably. Let $P$ denote the orthogonal projection of $L^{2}\left([0,2 \pi)\right.$ ) onto $H^{2}$ (regarded as a subspace of $\left.L^{2}\right)$. For an analytic function $\varphi$ in $H^{\infty}$ we use the notation $\tilde{\varphi}$ to denote the analytic function in $H^{\infty}$ whose $n$th Taylor coefficient is the complex conjugate of that of $\varphi$.

We shall now quote some relevant facts from [7]. (These are contained in the proof of Theorem 1.1 of [7].)

(I) For $\varphi$ in $H^{\infty}$ and $g$ in $H^{2}(\tau)$ define $T_{\bar{\varphi}} g=P(\bar{\varphi} g)$. Then $T_{\bar{\varphi}}$ is a bounded operator from $H^{2}(\tau)$ into itself and $\left\|T_{\bar{\varphi}}\right\| \leqq\|\varphi\|_{\infty}$.

(II) If $\varphi$ is a polynomial, $T_{\bar{\varphi}}=\tilde{\varphi}(B)$.

(III) If $\left\{\varphi_{n}\right\}$ is a sequence of polynomials and $\varphi$ is an element of $H^{\infty}$ such that $\left\{\left\|\varphi_{n}\right\|_{\infty}\right\}$ is bounded and such that $\varphi_{n}\left(e^{i \theta}\right) \rightarrow \varphi\left(e^{i \theta}\right)$ for a.e. $\theta$ in $[0,2 \pi)$, then for every $g$ in $H^{2}(\tau)$ a subsequence of $\left\{T_{\bar{\varphi}_{n}} g\right\}$ converges weakly to $T_{\bar{\varphi}} g$.

The operators $B$ and $S$ are both completely nonunitary contractions (in fact, $B^{n} \rightarrow 0$ strongly as $n \rightarrow \infty$ ). So we can make use of the functional calculus of Sz.-Nagy and Foias [13, Chapter 3]. If the sequence $\left\{\varphi_{n}\right\}$ in $H^{\infty}$ and the element $\varphi$ in $H^{\infty}$ are such that $\left\{\left\|\varphi_{n}\right\|_{\infty}\right\}$ is bounded and $\varphi_{n}\left(e^{i \theta}\right) \rightarrow$ $\varphi\left(e^{i \theta}\right)$ a.e. in $[0,2 \pi), \varphi_{n}(B) \rightarrow \varphi(B)$ in the strong operator topology [13, Theorem 2.1, p. 114 of the English translation]. By the observations (II) and (III) above we have the conclusion $\tilde{\varphi}(B)=T_{\bar{\varphi}}$. By the general results about the functional calculus of Sz.-Nagy and Foias $\tilde{\varphi}(B)^{*}=\varphi(S)$. In particular, we have $M_{\varphi}=\varphi(S)$ for every polynomial $\varphi$. By a direct verification we can prove that $T_{\bar{\varphi}}^{*}=M_{\varphi}$. Thus $M_{\varphi}=\varphi(S)$. Once again by the functional calculus, $M_{\varphi_{n}} \rightarrow M_{\varphi}$ in the strong operator topology if a sequence $\left\{\varphi_{n}\right\}$ in $H^{\infty}$ converges to $\varphi$ in $H^{\infty}$ boundedly and pointwise a.e. on the unit circle. This completes the proof of the Lemma.

Proof of Theorem 1. Let $e_{0}$ be the sequence $(1,0,0, \cdots)$. It suffices to prove that $e_{0}$ can be approximated in $l^{2}(\rho)$ by a finite linear combination of right translates of $a$. Write $f_{a}$ in the form $\varphi / \psi$ where $\varphi$ and $\psi$ are in $H^{\infty}$ and $\varphi$ is outer. Then $\psi f_{a}=\varphi$. Let $\alpha$ and $\beta$ be the sequences of Fourier coefficients of $\varphi$ and $\psi$, respectively. Then $\alpha=\beta * a$. By Beurling's theorem and the fact that $\varphi$ is outer, $e_{0}$ can be approximated by a finite linear combination of the right translates of $\alpha$ in the norm of the space $l^{2}$ (i.e. the space $l^{2}(\rho)$ with $\rho(n)=1$ for all $\left.n\right)$. Since our weight sequence $\{\rho(n)\}$ is decreasing, the same approximation works in the space $l^{2}(\rho)$. Thus, given $\varepsilon>0$, we can find a sequence $\gamma$ with only finitely many nonzero entries such that

$$
\left\|\beta * a * \gamma-e_{0}\right\|<\varepsilon
$$

where the norm above is the norm of $l^{2}(\rho)$. To complete the proof of the 
theorem, it suffices to find a sequence $\gamma^{\prime}$ with only finitely many nonzero entries such that

$$
\left\|\beta * a * \gamma-\gamma^{\prime} * a * \gamma\right\|<\varepsilon .
$$

This can be done easily by appealing to the lemma. Choose a sequence $\left\{\psi_{n}\right\}$ of polynomials such that $\psi_{n}\left(e^{i \theta}\right) \rightarrow \psi\left(e^{i \theta}\right)$ a.e. on $[0,2 \pi)$ and such that $\left\{\left\|\psi_{n}\right\|_{\infty}\right\}$ is bounded. We will simply choose $\gamma^{\prime}$ to be the sequence of coefficients of $\psi_{n}$ for a sufficiently large $n$.

The following corollary arose from a conversation with Allen Shields. It has been proved by de Leeuw and Rudin [6] when the weights are all equal to one.

COROLlary 1. Let $\{\rho(n)\}$ be a weight sequence satisfying the conditions of Theorem 1. If $a \in l^{2}(\rho)$ is such that $f_{a}$ has positive real part, then a is cyclic for the forward shift $S$ on $l^{2}(\rho)$.

Proof. Since $\operatorname{Re} f_{a}>0$, the same is true for $\operatorname{Re}\left(1 / f_{a}\right)$. It follows that $1 / f_{a}$ is in $H^{p}$ for $0<p<1$. (See [4, Theorem 3.2, p. 34].) It is also well known that $H^{p}$ is contained in $N^{+}[4$, p. 26]. Now the corollary follows from Theorem 1.

By the same kind of argument we can prove a more general result. (See [8, Theorem 4.4.9, p. 77].)

COROLlaRY 2. Let $\{\rho(n)\}$ be a weight sequence satisfying the condition of Theorem 1. If for an element a in $l^{2}(\rho)$ the function $f_{a}$ has the form $g^{k}$, where $k$ is a positive integer and $g$ is an analytic function in the unit disk with positive real part, then a is a cyclic vector for the forward shift $S$ on $l^{2}(\rho)$.

3. A problem of Aharonov, Shapiro and Shields. After an earlier version of this paper was written, a preprint of the paper [1] by Aharonov, Shapiro and Shields was made available to the author. In that paper the authors study weakly invertible elements in the Bergman space of squaresummable analytic functions in the unit disk. In the notations and terminology of the present paper, this is the study of cyclic vectors for the forward shift in the space $l^{2}(\rho)$ with $\rho(n)=(1+n)^{-1}$. We shall denote this space by $A_{1}$. More generally, let $A_{\varepsilon}$ denote the space $l^{2}(\rho)$ with $\rho(n)=$ $(1+n)^{-\varepsilon}$, where $\varepsilon>0$. On the basis of the main result of [1] and earlier results of Shapiro, the following question was raised in [1].

QUESTION. Let a be a sequence in $A_{\varepsilon}$ such that there exist positive constants $c$ and $k$ (depending on $a$ ) such that the inequality

$$
\left|f_{a}(z)\right| \geqq c(1-|z|)^{k}
$$

holds in $\{z:|z|<1\}$. Does it follow that a is a cyclic vector for the forward shift in $A_{\varepsilon}$ ? 
Theorem 2 stated below can be used to give a partial answer to this question. This theorem can be proved by essentially the same argument used to prove Theorem 1 .

THEOREM 2. Let $\rho$ be a weight sequence satisfying the conditions of Theorem 1. Let a be a vector in $l^{2}(\rho)$ such that $f_{a}=g f_{b}$, where $g^{-1} \in N^{+}$and $b$ is a cyclic vector for the forward shift on $l^{2}(\rho)$. Then $a$ is cyclic for the forward shift on $l^{2}(\rho)$.

THEOREM 3. Let a be an element of $A_{\varepsilon}(\varepsilon>0)$ such that $f_{a}$ is in the Hardy class $H^{p}$ for some $p>0$. If $f_{a}$ satisfies (6), then a is a cyclic vector for the forward shift on $A_{\varepsilon}$.

Proof. $f_{a}$ can be factored uniquely in the form $f_{a}=u F$ where $u$ is inner and $F$ is in $H^{p}$ and is outer [4, p. 24]. It can be seen easily that $F^{-1}$ is in the class $N^{+}$. Since $F$ is $H^{p}$, there exists a constant $M$ such that $|F(z)| \leqq$ $M(1-|z|)^{-1 / p}$ (see $[4$, p. 36]). Combining this with the assumption that $f_{a}$ satisfies an inequality of the type (6), we see that $u$ itself satisfies an inequality like (6). By Lemma 4 of [9] this implies that the sequence of Fourier coefficients of $u$ is cyclic in $A_{\varepsilon}$. Now Theorem 3 follows from Theorem 2.

RemarK. For the case $p \geqq 1$, Theorem 3 has been obtained earlier by Shapiro [11].

ACKNOWLEDGEMENTS. The main theorem of the paper had been proved earlier by Henry Helson and the author for a special weight sequence. The author wishes to thank Professor Helson for his kind permission to make use of some of his ideas in writing this paper. The author would also like to mention that Theorem 1.1 of [7] has been obtained independently, but by a different method by B. I. Korenbljum and V. M. Faǐvyševskiř [Ukrain. Mat. Ž. 24 (1972), 692-695].

\section{BIBLIOGRAPHY}

1. D. Aharonov, H. S. Shapiro and A. L. Shields, Weakly invertible elements in the space of square-summable holomorphic functions (to appear).

2. A. Beurling, On two problems concerning linear transformations in Hilbert space, Acta. Math. 81 (1948), 17pp. MR 10, 381.

3. - A critical topology in harmonic analysis on semigroups, Acta Math. 112 (1964), 215-228. MR 29 \#6255.

4. P. L. Duren, Theory of $H^{p}$ spaces, Pure and Appl. Math., vol. 38, Academic Press, New York, 1970. MR 42 \#3552.

5. P. R. Halmos, A Hilbert space problem book, Van Nostrand, Princeton, N.J., 1967. MR 34 \#8178.

6. K. deLeeuw and W. Rudin, Extreme points and extremum problems in $H_{1}$, Pacific J. Math. 8 (1958), 467-485. MR 20 \#5426. 
7. M. Rabindranathan, Toeplitz operators and division by inner functions, Indiana Univ. Math. J. 22 (1972), 523-529.

8. W. Rudin, Function theory in polydisks, Benjamin, New York, 1969. MR 41 \#501.

9. H. S. Shapiro, Weakly invertible elements in certain function spaces, and generators in $l_{1}$, Michigan Math. J. 11 (1964), 161-165. MR 29 \#3620.

10. - Weighted polynomial approximation and boundary behaviour of analytic functions, Contemporary Problems in Theory Anal. Functions (Internat. Conf., Erevan, 1965), "Nauka," Moscow, 1966, pp. 326-335. MR 35 \#383.

11. - Some remarks on weighted polynomial approximations of holomorphic functions, Mat. Sb. 73 (115) (1967), 320-330=Math. USSR-Sb. 2 (1967), 285-294. MR 36 \#395.

12. A. L. Shields and L. J. Wallen, The commutants of certain Hilbert space operators, Indiana Univ. Math. J. 20 (1970-71), 777-788. MR 44 \#4558.

13. B. Sz.-Nagy and C. Foiaș, Analyse harmonique des opérateurs de l'espace de Hilbert, Masson, Paris; Akad. Kiadó, Budapest, 1967; English transl., North-Holland, Amsterdam; American Elsevier, New York; Akad. Kiadó, Budapest, 1970. MR 37 \#778; 43 \#947.

Department of Mathematics, University of Michigan, Ann Arbor, Michigan 48104

Current address: Mathematical Reviews, 611 Church Street, Ann Arbor, Michigan 48104 